\title{
Comunicação efetiva entre o Centro Cirúrgico e a Unidade de Terapia Intensiva
}

\author{
Effective communication between surgical center and intensive care unit \\ Comunicación eficaz entre el Centro Quirúrgico y la Unidad de Cuidados Intensivos
}

\author{
Cristina Silva Sousa ${ }^{1}$, Regina Claudia da Silva Souza ${ }^{2}$, Maria Carolina Gonçalves ${ }^{3}$, Tania Regina Zeni Diniz ${ }^{4}$, \\ Ana Lucia Silva Mirancos da Cunha ${ }^{5}$
}

\begin{abstract}
RESUMO: Objetivo: construir um instrumento para a comunicação efetiva durante a passagem de plantão entre o Centro Cirúrgico e a Unidade de Terapia Intensiva. Método: relato de experiência, sobre a construção do impresso em quatro fases: brainstorming do grupo; confecção do impresso; teste-piloto; reformulação das informações e finalização do impresso. Resultados: foi elaborado um impresso com informações sobre pré-operatório (história prévia e antecedentes clínicos e cirúrgicos), intraoperatório (intercorrências, informação transfusional, antibiótico profilático, intubação difícil) e pós-operatório (condição de via aérea, drenos, sondas, curativos, medidas de prevenção de trombose, analgesia e transporte). Conclusão: o impresso proporcionou segurança e facilidade para transferência das informações para a unidade de destino e facilitou o planejamento da assistência de enfermagem sendo bem aceito pelos componentes de ambas as equipes.
\end{abstract}

PALAVRAS-CHAVE: Enfermagem de Centro Cirúrgico. Troca de informações. Continuidade da assistência ao paciente. Comunicação.

ABSTRACT: Objective: to create an instrument for effective communication between the operating room and the intensive care unit during shift handover. Method: a descriptive study aiming to create a print form in four phases: group brainstorming; manufacture of the printed form; pilot test; reformulation of information and completion of the print. Results: elaboration of a form with information about the preoperative (background, clinical and surgical history), intraoperative (complications, transfusion information, antibiotic prophylaxis, difficult intubation) and postoperative (condition of the airways, drains, catheters, bandages, measures to prevent thrombosis, analgesia, transportation) phases. Conclusion: the form provided safety and facility regarding information transfer to the destination unit and facilitated the planning of nursing care, being well accepted by the members of both teams.

KEYWORDS: Operating room nursing. Information exchange. Continuity of patient care. Communication.

RESUMEN: Objetivo: construir un formulario que sirva como instrumento para la comunicación eficaz durante el cambio de turno entre el Centro Quirúrgico y la Unidad de Cuidados Intensivos. Método: relato de experiencia sobre la construcción del formulario en cuatro fases: brainstorming del grupo; confección del formulario; prueba piloto; reformulación de la información y finalización del formulario. Resultados: se elaboró un formulario con informaciones sobre preoperatorio (historia previa y antecedentes clínicos y quirúrgicos), intraoperatorio (intercurrencias, información transfusional, profilaxis antibiótica, intubación difícil) y postoperatorio (condición de la vía aérea, drenajes, sondas, vendas, analgesia, transporte y medidas para prevenir la trombosis). Conclusión: el formulario proporcionó seguridad y facilidad a la hora de transferir la información a la unidad de destino, y facilitó la planificación de la asistencia de enfermería, por lo que fue bien aceptado por los miembros de ambos equipos.

PALABRAS CLAVE: Enfermería de Centro Quirúrgico. Intercambio de información. Continuidad de la atención al paciente. Comunicación.

${ }^{1}$ Enfermeira. Mestre em Enfermagem na Saúde do Adulto. Escola de Enfermagem. Universidade de São Paulo (EEUSP). Enfermeira Assistencial do Centro Cirúrgico.

Rua Professora Carolina Ribeiro, 20 apto. 54. Vila Mariana. CEP 04116-020. São Paulo, SP, Brasil.

Telefone: (11) 5083-3007/99601-7972. E-mail: crissousa@usp.br

${ }^{2}$ Enfermeira. Mestre em Enfermagem na Saúde do Adulto. Universidade de Guarulhos (UNG). Enfermeira Assistencial da Unidade de Terapia Intensiva.

Rua Frei Caneca, 239 apto. 53. CEP 01307-001. Consolação. São Paulo,SP, Brasil.

Telefone: (11) 97274-6161. E-mail: rclaudiasouza@uol.com.br

${ }^{3}$ Enfermeira. Especialista em Centro Cirúrgico, Recuperação Pós-Anestésica e Central de Materiais Esterilizados. Faculdade de Medicina. São José do Rio Preto (FAMERP).

Alameda Joaquim Eugenio de Lima, 712 apto. 903. Jardim Paulista. CEP 01403-000. São Paulo, SP, Brasil.

Telefone: (11) 3155-0749/98120-5018. E-mail: carolgonbr@ bol.com.br

${ }^{4}$ Enfermeira. Especialista em Centro Cirúrgico, Recuperação Pós-Anestésica e Central de Materiais Esterilizados. Associação Brasileira de Enfermeiros de Centro Cirúrgico

(SOBECC). Enfermeira Líder do Centro Cirúrgico.

Rua João Rodrigues Machado 28 apto. 33. Brooklin. CEP 04707-070. São Paulo, SP, Brasil.

Telefone: (11) 5181-9972. Email: trzeni@gmail.com

${ }^{5}$ Enfermeira. Mestre em Enfermagem na Saúde do Adulto. Escola de Enfermagem. Universidade de São Paulo (EEUSP). Enfermeira Coordenadora.

Rua Adma Jafet, 91. Bela Vista. CEP 01308-050. São Paulo, SP, Brasil.

Telefone: (11) 3155-0749/99399-5484. E-mail: ana.mirancos@ hsl.org.br 


\section{Introdução}

O início das atividades de enfermagem ocorre posteriormente à passagem de plantão, que requer do profissional Enfermeiro habilidade teórica, de práticas e de relacionamento interpessoal, enfatizando a comunicação e a gestão ${ }^{1}$. Nesse processo, diferentes formas de comunicação podem ser adotadas, sendo a verbal e a escrita as mais comuns, com destaque para a primeira.

$\mathrm{Na}$ assistência de enfermagem, o método de comunicação verbal entre o Centro Cirúrgico e a Unidade de Terapia Intensiva ocorre frequentemente por telefone. Interferências, como ruídos, outros profissionais chamando atenção e conversas paralelas podem ocasionar falhas na transmissão da informação.

Essas falhas na comunicação podem trazer prejuízos diretos para a assistência prestada ao paciente. A dificuldade de interação e a precária comunicação entre as equipes de enfermagem de origem e de destino para o paciente contribuem para o aumento significativo de complicações, que podem ser iniciadas no transporte intra-hospitalar e persistir durante a permanência do paciente na unidade, prolongando sua recuperação².

Nota-se que, durante a passagem de plantão, Enfermeiros costumam transmitir informações sobre suas ações e sobre os tratamentos recebidos pelos pacientes naquele período. Entretanto, vem se discutindo que deveriam ser transmitidas informações acerca das necessidades do paciente e não somente sobre as atividades que a enfermagem realiza, facilitando o planejamento da assistência ${ }^{3}$.

A passagem de plantão do Centro Cirúrgico para a Unidade de Terapia Intensiva tem o intuito de transmitir informações que direcionam o cuidado pós-operatório. Em algumas instituições, essa comunicação ainda não está presente e o Enfermeiro da Unidade de Terapia Intensiva recebe o paciente sem qualquer informação do intraoperatório, tendo de buscá-las no prontuário - o que demanda tempo e atrasa o planejamento do cuidado.

Essa atividade, pensada como instrumento básico e rotina que integra o trabalho de enfermagem, era uma inquietação do grupo quanto a comunicação, relacionamento interpessoal e trabalho em equipe dessas unidades críticas. É imprescindível que, nesse momento de transferência do paciente, as informações sejam precisas, claras e corretas, uma vez que, quando omitidas, imprecisas ou mal interpretadas, podem levar a falhas na assistência².

A fim de evitar possíveis falhas de comunicação e com o intuito de sistematizar a passagem de plantão de enfermagem, este estudo tem por objetivo construir um impresso que sistematize a passagem de plantão de enfermagem entre o Centro Cirúrgico e a Unidade de Terapia Intensiva.

\section{Método}

Estudo descritivo e analítico, do tipo relato de experiência, que tem como meta a construção de um impresso para sistematizar a passagem de plantão entre o Centro Cirúrgico e a Unidade de Terapia Intensiva. O estudo é um relato da experiência dos profissionais que construíram um protocolo para adequação de rotina de um hospital geral privado e filantrópico, localizado no município de São Paulo.

A construção do impresso foi idealizada por um grupo de Enfermeiros do Centro Cirúrgico e da Unidade de Terapia Intensiva adulto, que desejava melhorar o processo de comunicação entre essas unidades e assegurar a troca de informações de forma sistematizada e segura.

O processo de construção do impresso para a passagem de plantão teve quatro fases: 1) comunicação intersetorial; 2) confecção do impresso; 3) teste-piloto; 4) finalização do impresso.

\section{Primeira fase: comunicação intersetorial}

O primeiro passo para construção do impresso foi propor um encontro entre os Enfermeiros das unidades (Centro Cirúrgico e Unidade de Terapia Intensiva adulto), para discutir as informações inseridas no impresso. O objetivo foi compreender a necessidade dos profissionais da Unidade de Terapia Intensiva e encontrar uma forma de operacionalizar o processo de comunicação, para que os profissionais das respectivas unidades ficassem satisfeitos com o resultado.

O convite para o encontro foi formalizado por e-mail entre os Enfermeiros que participaram do grupo, com determinação do local e do horário para discussão. O local escolhido foi a sala de reuniões do Centro Cirúrgico.

\section{Segunda fase: confecção do impresso}

A construção do impresso foi realizada após a primeira fase. Os dados foram inseridos no Microsoft Word 2007® por uma Enfermeira do grupo. O formato das informações contempla espaço para descrição e itens em formato de checklist com o intuito de facilitar o preenchimento.

A validação das informações foi realizada por meio da leitura do impresso e da sua devolução com correções, entre os Enfermeiros do grupo.

\section{Terceira fase: teste-piloto}

Após a validação, foi proposto um teste-piloto para avaliar a viabilidade do processo e a necessidade de refinamento do impresso. Foi determinado um período de 15 dias para avaliação, sendo que, nesse período, em todos os plantões, a comunicação entre as unidades foi realizada por meio do impresso. Após o preenchimento pelo Enfermeiro do Centro Cirúrgico, o Técnico de Enfermagem júnior do Centro Cirúrgico leva o impresso ao Enfermeiro da Unidade de Terapia Intensiva responsável pelo cuidado pós-operatório do paciente a ser transferido. 


\section{Quarta-fase: finalização do impresso}

Após o teste-piloto, algumas alterações no impresso foram inseridas pelos Enfermeiros do Centro Cirúrgico - monitorização da pressão intracraniana, curativo com sistema a vácuo e acréscimo de espaço para antecedentes clínicos e cirúrgicos - visando a melhorar a comunicação. Essas informações não constavam no instrumento e na ocorrência de pacientes que requeriam a informação, sendo esta escrita no rodapé do impresso.

\section{Resultados}

\section{Primeira fase: comunicação intersetorial}

No encontro marcado, o grupo foi composto por duas Enfermeiras da Unidade de Terapia Intensiva e três Enfermeiras do Centro Cirúrgico, que representaram suas unidades. A ideia do impresso foi apresentada e um esboço do conteúdo foi apresentado. Nesse momento, foram expostas as necessidades das Enfermeiras da Unidade de Terapia Intensiva sobre as informações necessárias na assistência pós-operatória. As informações foram divididas em:

- Pré-operatório: história prévia e antecedentes;

- Intraoperatório: intercorrências, antibiótico profilático, manejo de via aérea;

- Pós-operatório: medidas de prevenção de trombose e analgesia.

Foi adicionado também o contato direto do Enfermeiro responsável pelo paciente no Centro Cirúrgico para facilitar o contato em caso de dúvidas.

Discutiu-se a estratégia de entrega do impresso e foi estabelecido o tempo de 30 minutos de antecedência para a transferência do paciente. Esse tempo é o mínimo necessário para o preparo da Unidade de Terapia Intensiva e para o planejamento da assistência. Optou-se por não utilizar a comunicação verbal das informações contempladas no impresso; ou seja, o impresso substituiu a comunicação verbal realizada anteriormente.

\section{Segunda fase: confecção do impresso}

O impresso consiste em uma folha A4, com preenchimento frente e verso. Foram criadas três grandes aéreas:

- Pré-operatório: com duas lacunas descritivas, história prévia e antecedentes;

- Intraoperatório: com cinco itens, intercorrências, informação transfusional, antibiótico profilático, manejo de via aérea e tempo de circulação extracorpórea;

- Pós-operatório: com itens que envolvem condições de via aérea; dispositivos de sondagem; acesso venoso; monitorização; curativos; drenos; infusão de líquidos ou drogas vasoativas; estratégias de profilaxia de trombose; prescrição de analgesia, e informações a respeito do transporte do paciente.
Após a impressão da primeira versão, o impresso foi enviado para os Enfermeiros do grupo para análise e correção. A validação se deu pela leitura dos itens pelos participantes e, se necessário, após essa etapa, fez-se a inclusão ou a retirada de informações. Não houve solicitações por parte dos Enfermeiros da Unidade de Terapia Intensiva, o que resultou em 100\% de aceite por parte dessa unidade. Para os Enfermeiros do Centro Cirúrgico, foi requerido o acréscimo de alguns itens na área de pós-operatório, como curativos, analgesia e drenos. As solicitações foram atendidas e o impresso foi submetido à reavaliação, obtendo $100 \%$ de aceite pelos Enfermeiros do Centro Cirúrgico.

\section{Terceira fase: teste piloto}

O teste do impresso ocorreu na primeira quinzena de outubro de 2012. Nos primeiros dias, notou-se que os profissionais da Unidade de Terapia Intensiva ficaram apreensivos com o novo método de passagem de plantão e retornavam a ligação para confirmar as informações.

Nesse primeiro momento, é compreensível a resistência ao novo: a mudança no processo de comunicação gerou dúvidas nos profissionais das duas unidades envolvidas.

Para assegurar a melhoria no processo, foi solicitado aos Enfermeiros do grupo que participaram da construção do impresso que auxiliassem na divulgação da informação. A mesma estratégia foi realizada no Centro Cirúrgico, cujos profissionais também foram orientados.

Ao fim do período determinado como piloto, os profissionais estavam mais receptivos com a nova estratégia e surgiram resultados positivos, como: facilidade no processo de transferência do paciente, sendo o impresso preenchido durante o intraoperatório pelo Enfermeiro responsável pela sala cirúrgica; sistematização das informações, na medida em que as informações se tornaram uniformes e atenderam às necessidades dos profissionais da Unidade de Terapia Intensiva; segurança na comunicação, pois evita falhas na comunicação entre os profissionais e a Enfermeira da unidade de destino pode consultar a informação sempre que necessário; facilitação do contato direto com o Enfermeiro do Centro Cirúrgico, pois há a divulgação do telefone corporativo do Enfermeiro do Centro Cirúrgico responsável pelo paciente, para contato em caso de dúvidas.

\section{Quarta-fase: finalização do impresso}

Na segunda quinzena de outubro de 2012, a estratégia de comunicação escrita para a passagem de plantão seguiu o processo proposto no teste-piloto e não foram necessárias alterações no procedimento ou no tempo de entrega do impresso.

O impresso foi colocado na pasta dos Enfermeiros do Centro Cirúrgico para garantir acesso ao documento por todos os profissionais. Na Unidade de Terapia Intensiva adulto, uma pasta com elástico foi colocada na sala dos 
Enfermeiros, centralizando a guarda dos formulários para uso em pesquisas posteriores.

As dificuldades vivenciadas durante o teste-piloto não foram vistas posteriormente. $\mathrm{O}$ processo de construção do impresso foi finalizado e instituído como rotina do procedimento de passagem de plantão para Unidade de Terapia Intensiva (Anexo 1).

\section{Discussão}

Em um cenário complexo, tornam-se indiscutíveis a necessidade e a importância de um sistema de informação que propicie aos Enfermeiros informações necessárias para o planejamento da assistência ${ }^{1}$. A comunicação configurase como um elemento essencial ao cuidado e pode ser entendida como alicerce de nossas relações interpessoais. Com definição complexa e formas variadas, deve-se buscar melhorar a compreensão e a interação entre os partícipes da comunicação $0^{4}$.

A Enfermeira do Centro Cirúrgico não tem somente responsabilidade para com seus pacientes no intraoperatório, mas para com as outras Enfermeiras, a fim de promover o cuidado de enfermagem coordenado e contínuo ${ }^{5}$. Dessa forma, deve transferir as informações sobre o intraoperatório de maneira fidedigna para a Enfermeira da unidade de destino, visando a auxiliar no planejamento da assistência no pós-operatório.

Um estudo de São Paulo (BR) ${ }^{2}$ sobre o transporte intrahospitalar destacou, entre os eventos adversos, a falta de conhecimento do profissional e a falha de comunicação. Ressalta-se a falta de conhecimento sobre o verdadeiro quadro clínico do paciente ou a ausência de informações básicas sobre o paciente, tais como idade, peso, diagnóstico, procedimento realizado, estabilidade hemodinâmica, padrão respiratório, acesso venoso e gotejamento de medicamentos.

Atualmente, em nossa instituição, a comunicação escrita é tida como uma prática rotineira na Unidade de Terapia Intensiva entre os turnos de trabalho. As informações acerca do paciente são deixadas por escrito entre os profissionais de turnos diferentes e têm-se mostrado eficazes, como parte integrante da assistência.

Não há estudos recentes que relatem a passagem de plantão escrita na enfermagem e poucas publicações retratam a passagem de plantão como estratégia de comunicação e sua importância para a assistência de enfermagem.

A continuidade do cuidado foi fator motivador para as Enfermeiras deste grupo. Notou-se diminuição de eventos adversos, falhas ou mal-entendidos na transmissão das informações durante a passagem de plantão. No entanto, os impactos no uso do impresso ainda não foram mensurados, devido ao pouco tempo de sua implantação.

Em um estudo multicêntrico em oito hospitais no sudeste de Michigan (USA) ${ }^{6}$, Enfermeiros em Unidades de Terapia Intensiva identificaram que melhorar as características do ambiente de assistência, como os fatores que interferem na comunicação, torna o processo mais efetivo. O resultado mostra que o ambiente tem uma influência direta e impactante no sistema de informação. Assim, estabelecer estratégias de comunicação escrita bem estruturadas é importante e contribui com resultados positivos.

Nesse mesmo estudo ${ }^{6}$, a falha na comunicação verbal entre Enfermeiros e Médicos foi responsável por 37\% de todos os erros. Outro estudo do Reino Unido (UK), com o objetivo de avaliar falhas no trabalho em equipe e na comunicação no pós-operatório de pacientes submetidos à cirurgia gastrointestinal, revelou $41 \%$ (105) de casos de falha de comunicação, sendo que, destes, $99 \%$ eram evitáveis. Entre as falhas de comunicação, destacaram-se a comunicação escrita pobre e a falta de comunicação verbal ${ }^{7}$.

Assim, a incorporação de uma cultura voltada para a segurança do paciente, com implantação de processos que assegurem essa meta e estejam focados em resultados esperados e eficazes, é desejável. O processo de construção da sistematização da passagem de plantão objetiva a segurança do paciente. $\mathrm{O}$ impresso pode assegurar que as informações do intraoperatório sejam recebidas de forma organizada e correta, sem perdas ocasionais por comunicação verbal em ambiente ruidoso, como a Unidade de Terapia Intensiva.

A comunicação interfere diretamente na qualidade da assistência prestada e é um recurso utilizado para o sucesso da liderança exercida pelo Enfermeiro. Uma relação de comunicação eficiente entre todos os membros da equipe multidisciplinar e de enfermagem contribui para que as interrelações profissionais estabelecidas no trabalho delimitem melhor se a assistência ao paciente será ou não humanizada4 ${ }^{4}$.

Otimizar o tempo é obrigatório aos profissionais de enfermagem para prover o cuidado direto ao paciente. Cabe ao Enfermeiro Assistencial do Centro Cirúrgico, levantar os dados importantes para o cuidado de pós-operatório imediato na Unidade de Terapia Intensiva e, com isso, garantir a continuidade do cuidado com segurança. A comunicação escrita demanda um desgaste menor de ambos os profissionais, já que eles não necessitam interromper suas atividades para troca de informações por telefone, evitando, assim, possíveis interferências.

A construção de um instrumento para sistematizar a passagem de plantão melhorou a comunicação entre duas unidades críticas e a promoção da continuidade do cuidado. Após a implantação do impresso, a Unidade de Terapia Intensiva pediátrica referiu interesse na aplicação do instrumento e novas necessidades devem ser levantadas para atender a essa unidade.

Uma das dificuldades vivenciadas durante a implantação do impresso foi a pouca receptividade da equipe de enfermagem na recepção das informações e dos pacientes; o novo fluxo de comunicação entre as unidades tornou necessárias novas orientações. É fundamental que pessoas inseridas nos sistemas de saúde vejam a comunicação e a relação interpessoal como fatores contribuintes para a segurança do paciente ${ }^{8}$. As falhas nesse processo estão 
intimamente relacionadas com a ocorrência de eventos adversos.

As vantagens observadas com o impresso foram o fluxo de comunicação seguro e eficaz, além da otimização do tempo dos profissionais pela agilidade do processo e a ocorrência de poucas interferências, como ruídos e interrupções. Sabese que o processo comunicativo está inserido nas atividades do cotidiano dos profissionais de saúde e se constitui em um fenômeno importante, pois facilita o desempenho das funções desses profissionais em relação ao paciente e à equipe. Portanto, é essencial o conhecimento por parte da equipe dos mecanismos que facilitam esse processo ${ }^{9}$.

A continuidade do cuidado é o princípio da prática profissional da enfermagem e a comunicação sobre as condições e necessidades do paciente entre os profissionais da saúde é fundamental para se alcançar uma assistência contínua e eficaz.

\section{Considerações finais}

A comunicação entre os profissionais de saúde torna-se essencial ao cuidado do paciente crítico. Por meio dessa ferramenta, é possível garantir a eficácia do cuidado, sua continuidade e o planejamento adequado.

Acreditamos que o impresso não significou aumento de burocracia e, sim, aumento da segurança para o paciente. Com esse instrumento, a Enfermeira da Unidade de Terapia Intensiva pode adiantar o planejamento de cuidados, preparar melhor o leito do paciente e se programar nas atividades de seu turno de trabalho. Por sua vez, a Enfermeira do Centro Cirúrgico registra a informação necessária para a passagem de plantão e minimiza o tempo dispendido na troca de informações por telefone.

\section{Referências}

1. Feliciano Marques L, Santiago LC, Curitiba Felix V. A passagem de plantao como elemento fundamental no processo de cuidar em enfermagem: o perfil da equipe de enfermagem de um hospital universitário. Rev Pesqui Cuid Fundam. 2012;4(2):2878-82.

2. Almeida ACG, Neves ALD, Souza CLB, Garcia JH, Lopes JL, Barros ALBL. Transporte intra-hospitalar de pacientes adultos em estado crítico: complicações relacionadas à equipe, equipamentos e fatores fisiológicos. Acta Paul Enferm. 2012;25(3):471-6. http:// dx.doi.org/10.1590/S0103-21002012000300024

3. Dowding D. Examining the effects that manipulating information given in the change of shift report has on nurses' care planning ability. J Adv Nurs. 2008;33(6):836-46. http://dx.doi. org/10.1046/j.1365-2648.2001.01723.x

4. Broca PV, Ferreira MA. Equipe de enfermagem e comunicação: contribuições para o cuidado de enfermagem. Rev Bras Enferm. 2012;65(1):97-103. http://dx.doi.org/10.1590/ S0034-71672012000100014

5. Klein AS, Bitencourt JVOV, Dal Pai D, Wegner W. Registros de enfermagem no período perioperatório. Rev enferm UFPE on line 2011;5(5):1096-104. http://dx.doi.org/10.5205/ reuol.1302-9310-2-LE.0505201103

6. Manojlovich M, DeCicco B. Healthy work environments, nursephysician communication, and patients' outcomes. Am J Crit Care. 2007;16(6):536-43. PMid:17962497.

7. Symons NRA, Almoudaris A, Nagpal K, Vincent CA, Moorthy $\mathrm{K}$. Teamwork and communication failures in post-operative care. J Am Coll Surg. 2011;213(3):S111. http://dx.doi.org/10.1016/j. jamcollsurg.2011.06.260

8. Nagpal K, Arora S, Vats A, Wong HW, Sevdalis N, Vincent $\mathrm{C}$, et al. Failures in communication and information transfer across the surgical care pathway: interview study. BMJ Qual Saf. 2012;21(10):843-9. PMid:22773891. http://dx.doi.org/10.1136/ bmjqs-2012-000886

9. Machado EP, Haddad JGV, Zoboli E. A comunicação como tecnologia leve para humanizar a relação enfermeiro-usuário na atenção básica. Rev Bioethikos. 2010;4(4):447-52. 
Anexo 1. Instrumento para a passagem de plantão do CC e a UTI.

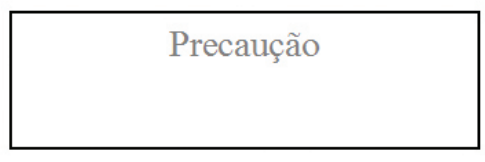

\section{Encaminhamento para UTI}

ALA _ BOX

Procedimento Cirúrgico:

Cirurgião:

\begin{tabular}{|c|c|}
\hline $\begin{array}{l}\text { História pregressa } \\
\text { da moléstia atual }\end{array}$ & \\
\hline Antecedentes & $\begin{array}{l}\square \text { HAS } \square \text { DM } \square \text { Cardiopatia } \square \text { Hipotireoidismo } \square \text { Insuficiência renal } \\
\square_{\text {DLP }} \square_{\text {AVE }} \square_{\text {Déficit }} \\
\text { Cirúrgicos: } \\
\square \text { Alergia } \_ \\
\text {Peso: } \quad \text { kg Altura: } \_\mathrm{cm}\end{array}$ \\
\hline \multirow[t]{5}{*}{ Intraoperatório } & Intercorrências: \\
\hline & $\begin{array}{l}\text { Transfusão: Concentrado de hemácias __unidades } \\
\text { Plasma Fresco___ unidades } \\
\text { Criopreciptado__ unidades } \\
\text { Aférese de Plaquetas__ unidades }\end{array}$ \\
\hline & IOT Difícil $\quad \square \operatorname{sim} \quad \square$ não \\
\hline & Antibiótico profilático. \\
\hline & Cirurgia Cardíaca: Tempo de CEC ___ Tempo de anóxia \\
\hline
\end{tabular}


Anexo 1. Continuação...

\begin{tabular}{|c|c|}
\hline Pós-operatório & $\square$ IOT $\square$ Traqueostomia $\square$ Nebulização $\square$ Cateter Oxigênio \\
\hline & $\square \mathrm{SNG} \square \mathrm{SNE}$ \\
\hline & Acesso central: $\square$ DL__ $\square \mathrm{TL} \_\square$ Port-a-cath__ $\square$ Picc \\
\hline & Acesso periférico: $\square$ MSD \\
\hline & Infusão: $\begin{aligned} & \square \text { RL } \square \text { Plasmalyte } \square \text { Gelafundim } \square \text { SF0,9\% } \\
& \square \text { Noradrenalina__mcg/kg } \square \text { Adrenalina__ mcg/kg } \\
& \square \text { Dobutamina _ mcg/kg } \square \text { Nipride__ mcg/kg } \\
& \square \text { Tridil__mcg/kg } \quad \square\end{aligned}$ \\
\hline & 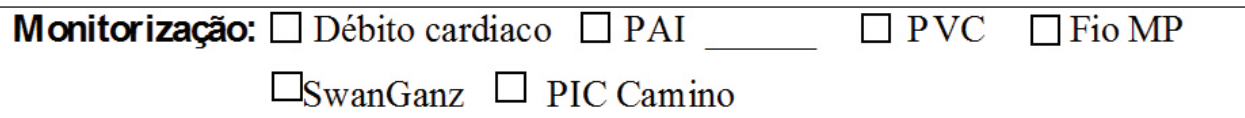 \\
\hline & Curativos: $\begin{aligned} & \square \text { Opsite } \square \text { Prineo } \square \text { Dermabond } \square \text { Gaze e Micropore } \square \text { VAC } \\
& \square \text { Enfaixamento } \quad \square \text { Steri-strip } \square \text { Hidrocoloide }\end{aligned}$ \\
\hline & Drenos: $\begin{aligned} & \square \text { JP } \\
& \square \text { Nelaton } \square \text { Blacke_ } \square \text { Bolsa de transferência } \square \text { Penrouse } \\
& \square \text { Pleural_ } \square \text { Mediastino } \square \text { Pigtail_ } \square \\
& \square \text { Gastrostomia } \square \text { Gastrojejunostomia } \\
& \square \text { Cistostomia } \square \text { Nefrostomia __ } \square \text { Ileostomia } \square \text { Colostomia } \\
& \square \text { SVD }\end{aligned}$ \\
\hline & Profilaxia de TEV: $\square$ meia elástica $\quad \square$ massageador $\square$ MMII $\square$ plantar \\
\hline & $\begin{aligned} \text { Analgesia: } & \square \text { Peridural } \square \text { Contínua } \square \text { Intermitente } \square \text { Dose única } \\
& \square \text { PCA Venosa } \\
& \square \text { Bloqueio de Plexo }\end{aligned}$ \\
\hline & $\begin{array}{l}\text { Transporte: } \\
\square \quad \text { Maca } \quad \square \quad \text { Cama } \square \text { Ventilador Savina } \square \text { Ventilador Oxilog }\end{array}$ \\
\hline
\end{tabular}

Enfermeira:

Celular: 